\title{
Designing a tote Quad copter, an Unmanned Aerial Vehicle (UAV)
}

\author{
K.Srinivasu ${ }^{1}$, A.Vijaya Shanthi ${ }^{2}$ \\ M. Tech, Electronics \& Instrumentation Department \& GITAM University, Gandhi Nagar Campus, Rushikonda, \\ Visakhapatnam, Andhrapradesh, India ${ }^{1}$ \\ Assistant Professor (M.E.), Electronics \& Instrumentation Department \& GITAM University, Gandhi Nagar Campus, \\ Rushikonda, Visakhapatnam, Andhrapradesh, India ${ }^{2}$
}

\begin{abstract}
The main concern of this paper is to notify the designing of a quad copter which is capable of carrying a payload and the camera, as it is used to determine the location of the dock. A quad copter is designed such that it can correctly determine the location of a target ground station while hovering and then land on the target. To deal with quad copter, weight is the main constrain which is important and play a predominant role in these unmanned aerial vehicles. So, from this paper we can also make the design of a quad copter with lightweight.
\end{abstract}

Keywords: Quad copter, Arduino nano, nRF24L01, Multiwii v2.5

\section{INTRODUCTION}

A quad copter is a quad rotor helicopter which is having the four motors, but it is entirely different whereas the lift force is produced by the four motors. The similarity exist between the helicopter and the quad copter is the vertical take-off and landing.

Quad copter is an ideal solution for many indoor and outdoor applications that presently jeopardize human or material safety.

These quad copters are controlled by using a remote control, for that reason they can be used in three area such as military to do spying on enemy camps, to avoid loss of man power these are unmanned aerial vehicles searching in place where the human cannot serve and recently in transportation of goods such as medicines.

A quad copter with four rotors as each rotor has its own significance in creating thrust, torque and direction. The propellers which create the thrust to the quad copter and the rotation of these motors has been fixed as clock wise for the left of front as well as right of rear and anti-clock wise for right of front as well as left of rear, now the clock wise rotors act as pullers and other two anti-clockwise rotors act as pushers. As consequence the resulting torque is zero.

Because of its unique design comparing to traditional helicopters, it allows a more stable platform and with the additional attachment of the camera to the quad copter in this project we can determine the location and guide for the dock with aerial photography.

The flight control of the quad copter can be divided into four types as throttle, yaw, pitch and roll.

And their function varies from each other by the specific control of each rotor as given in the figures here.

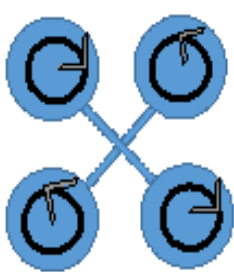

(a)

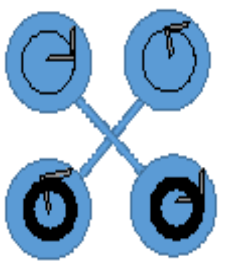

(c)

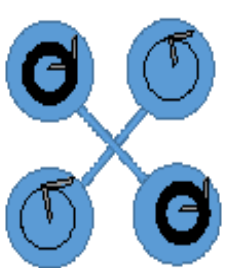

(e)

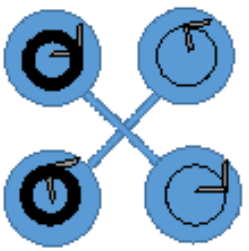

(g)

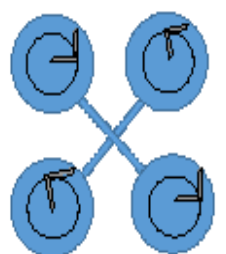

(b)

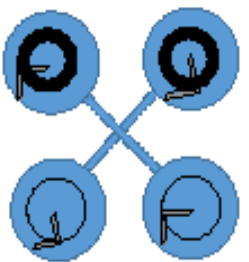

(d)

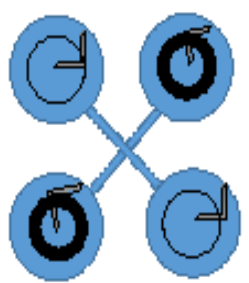

(f)

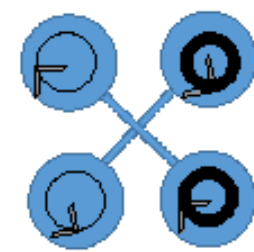

(h)
Fig.1 Different types of flight controls for quad copter are (a) throttle to move up, (b) throttle to lower, (c) to move forward, (d) to move backward, (e) to move counter clock wise yaw, (f) to move clock wise yaw, (g) to move right, (h) to move left. 


\section{HARDWARE REQUIREMENTS}

All projects have a set of requirements and a traditional quad copter has the requirement of four small rotors at each of the four corners. This has become the most common design because it's easy to control and keep stable in flight. However, using four small rotors isn't very efficient, and it severely limits maneuverability. The design of resonators, gyroscopes, accelerometers and actuators consider the effects of several physical phenomena on their operation.

\section{A) Frame}

The body of the quad copter which can be said that as frame of the quad copter is the main concern because we can increase the payload for it with lesser weight of the frame. And the frame of the copter can be constructed with different types of materials. The Q $450 \mathrm{~V} 3$ carbon fibre frame is used with the weight of 270 grams and it has inbuilt PCB used to connect ESCs.

\section{B) Arduino nano}

The Arduino nano has a number of facilities for communicating with a computer, another Arduino or other microcontrollers. It is a small, complete and breadboardfriendly board based on the ATmega328.

Arduino is necessary since the Pulse-width modulation (PWM) is a better controlling technique when dealing with power-to-inertial electrical devices, such as in the case of controlling BLDC motors. The PWM uses a digitallygenerated rectangular-pulse waveform to employ the switching between the supply and the load voltage.

\section{C) Camera with servo motor}

A camera is used to have a visual for docking which can be controlled with the servo motor.

\section{D) BLDC motor}

In quad copter four rotors with brushless motors are used to get high efficiency for less power and low weight. These are synchronous motors and to drive them, AC electric signal required driven by a DC electric source via ESC. A BLDC motor, which is specified as $2200 \mathrm{KV}$ and weights up to 47 grams is used for producing a good thrust to lift the quad copter.

\section{E) ESC (Electronic Speed Controller)}

ESCs run with DC voltage and require PWM signal controllers for BLDC motors. It is important to utilize only the PWM signal controllers when connecting the motors to the Arduino. And 20A ESC was used in order to avoid the fluctuations in current from battery.

The ESC will provide the necessary command tool and communication for the instructions given to the motors regarding their change of speed.

The ESC is essential in allowing the control systems representative to control the variance of the speed and altitude that the quad copter will undergo.
Generally, the ESC accepts a nominal servo input of $50 \mathrm{~Hz}$ PWM signal with a varying pulse width from $1 \mathrm{~ms}$ to $2 \mathrm{~ms}$. This is important during the controls command for the calculating and ultimately the deciding process of which speed value is high enough to make the quad copter hover, and which speed is high enough to make it fly up high. At a $1 \mathrm{~ms}$ width pulse, the ESC turns off the DC motor attached to its output. At a $1.5 \mathrm{~ms}$ pulse-width input signal, the ECS runs a $50 \%$ duty cycle output signal which is interpreted at half of the maximum threshold speed for the motors. Lastly, at a $2.0 \mathrm{~ms}$ input signal, the ESC runs at $100 \%$ duty cycle and provides the motors $100 \%$ of its maximum speed.

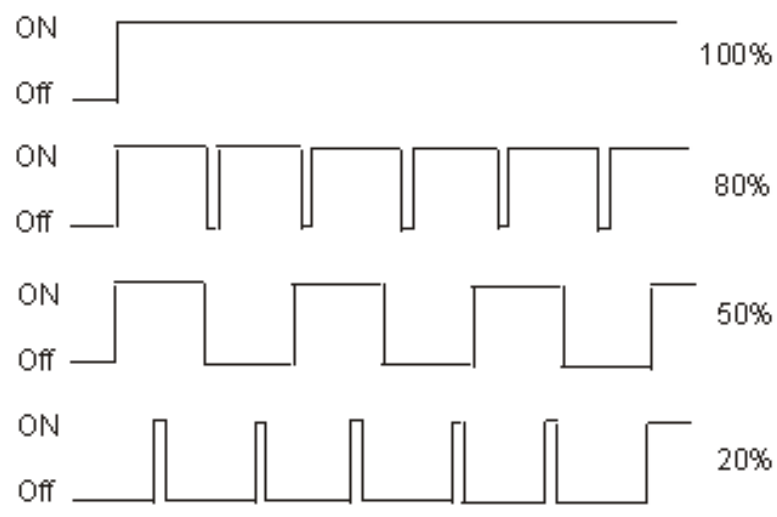

ON

Off

Fig.2 PWM duty cycle

\section{F) Propeller}

Propellers are used to generate the thrust for the quad copter hover or lift. These are in different variants which are classified based on their diameter and pitch by which they travel. And for Quad copter four of them were mounted on the BLDC motors. Four of the $(10 * 4.5)$ propellers were used.

\section{G) Battery}

As for the power source of the quad copter, LiPo Battery is better to use as it is light. The $1300 \mathrm{mAh}$ LiPo battery can be used with 3SP1 batteries which means three cells connected in series as one parallel, which gives $11.1 \mathrm{~V}$.

\section{H) nRF module}

The nRF $24 \mathrm{LO} 1$ is a transceiver with $2.4 \mathrm{GHz}$ operating frequency and also suitable for ultra-low power wireless applications like quad copter and other aerial vehicles.

To design a radio system with this module, we use Arduino nano as microcontroller and it was based on packet communication and supports various modes from manual operation to advanced autonomous protocol operation.

Two pairs of Arduino and nRF module were used for communication as one pair is at receiver i.e., at the quad copter and the other one is at transmitter. 
INTERNATIONAL JOURNAL OF INNOVATIVE RESEARCH IN ELECTRICAL, ELECTRONICS, INSTRUMENTATION AND CONTROL ENGINEERING Vol. 3, Issue 4, April 2015

I) MultiWii v2.5

It is a compatible flight controller which is one of the least expensive multi-rotor flight controllers. Today the MultiWii project is a very powerful open source software that keeps getting better with each revision release and is compatible with a variety of self-flight controllers and sensors.

This flight controller has specifications with ATmega 2560 microcontroller; MPU6050, a 6 axis gyroscope and accelerometer; HMC5883L, a 3 axis digital magnetometer. It can control up to 8 axis motor output.

In order to communicate with the MultiWii board, we must download and install the correct USB to serial drivers $(\mathrm{VCP}=$ Virtual Com Port $)$.

\section{DESIGNING THE QUAD COPTER}

The quad copter's designing is mainly divided into two parts as the first part is to construct the main body of the quad copter and then the controller part which was used to control the quad copter.

\section{Designing a receiver for quad copter}

The quad copter hardware part is the embedding of the components as mentioned above. The carbon fibre is the light weight and strong enough to handle thrust which has been produced by the propellers which were inclined on BLDC motors. In order to avoid the fluctuations of the body in air the $\mathrm{X}$ shaped frame is made.

The nRF24L01 which acts as receiver is connected to Arduino with the input power of $3.3 \mathrm{v}$ and it gives the data from transmitter to control the motors. The BLDC motors which were connected to ESC so as to get the AC power supply from DC battery i.e., the DC from LiPo battery is converted into AC by ESC to drive the motors.

MultiWii is used to control the four motors at the same time with the help of Arduino nano. The camera which can also be controlled by Arduino with the help of servo motor. For the software part we use Arduino software, open source for Arduino as well as MultiWii can be configured with it such that the board selection, the type of flight control used and ESC which were used for the quad copter are modified in the program and this has to be uploaded to the MultiWii board.

MultiWii GUI is used to determine the working procedure 2 of quad copter i.e., its flight mode, RC transmitter values, ESC values, model in 3D display, horizon, compass, MultiWii exponential \& rate as well as multiWii sensor values with graph.

Before performing the test for quad copter this MultiWii configuration is very useful as it is connected to COM port and the calibrations are calculated regarding accelerometer then it is written into the port. The testing for throttle, yaw, pitch and roll can also be done using this.

The quad copter can be configured as arm and disarm i.e., on and off switches. By making throttle to low and yaw to high quad copter can be armed, as well as by making throttle and yaw to low quad copter can be disarmed.

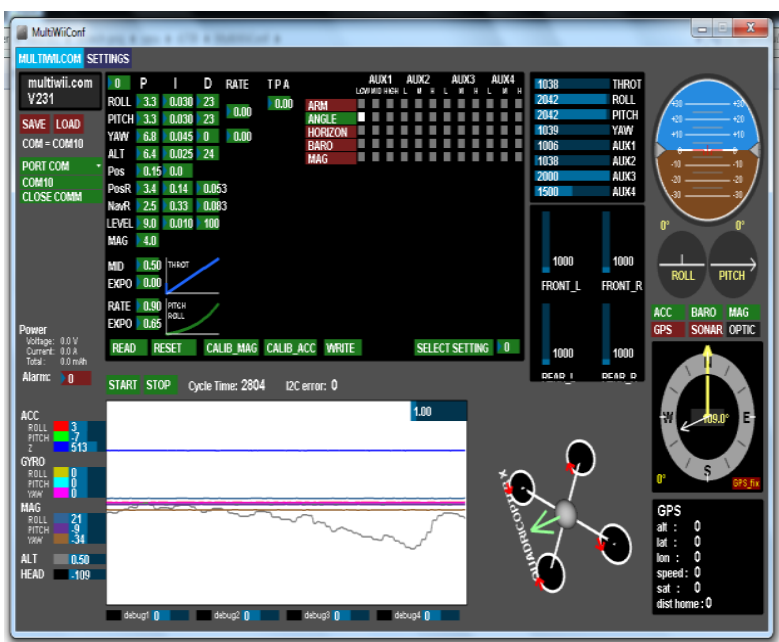

Fig. 3 MultiWii configuration interface (GUI)

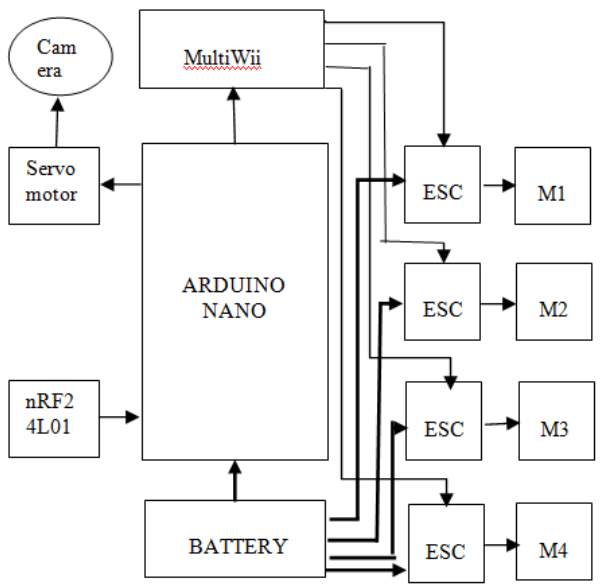

Fig.4 Block diagram of receiver for quad copter

The block diagram shown above indicates the functioning of the quad copter at receiver which consists of mainly the first pair of Arduino nano and nRF24L01 as the nRF module receives the signal from transmitter which has operated by the user. The controlling is done by MultiWii as it can change the rotation of the motors from Arduino with the help of ESC. And the BLDC motors were represented as M1, M2, M3 and M4 in the above block diagram.

2 .

Designing a transmitter for quad copter

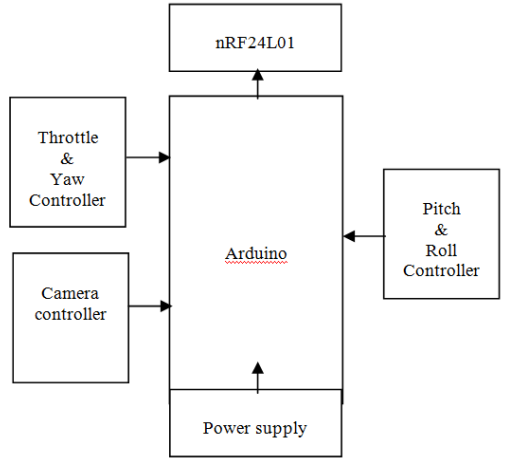

Fig. 5 Block diagram of Transmitter for Quadcopter 
The transmitter part consists of the second pair of Arduino vehicle. The complete design of this quad copter would and another nRF module which was used for transmitting resemble the stabilized and light weight quad copter with the data to quad copter. The data which was transmitted is some payload as well as for safe landing with the help of an analogue signal produced by joysticks for throttle, yaw, camera. Thus this quad copter which has been designed, pitch and roll that is by varying the potentiometer of the can be playing a predominant role in transportation of joystick.

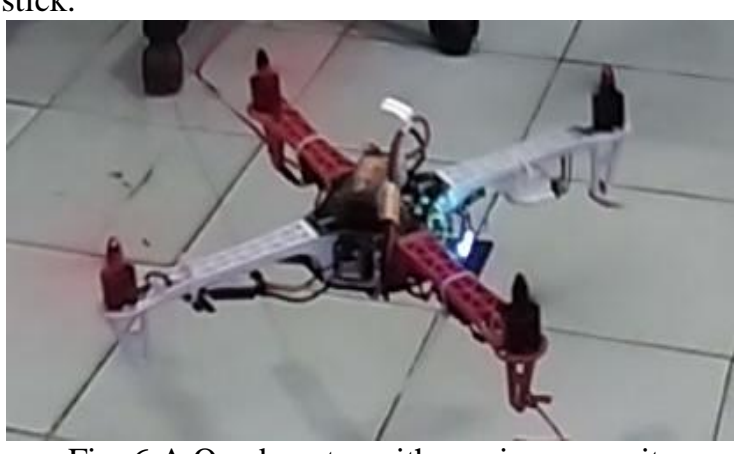

Fig. 6 A Quad copter with receiver upon it.

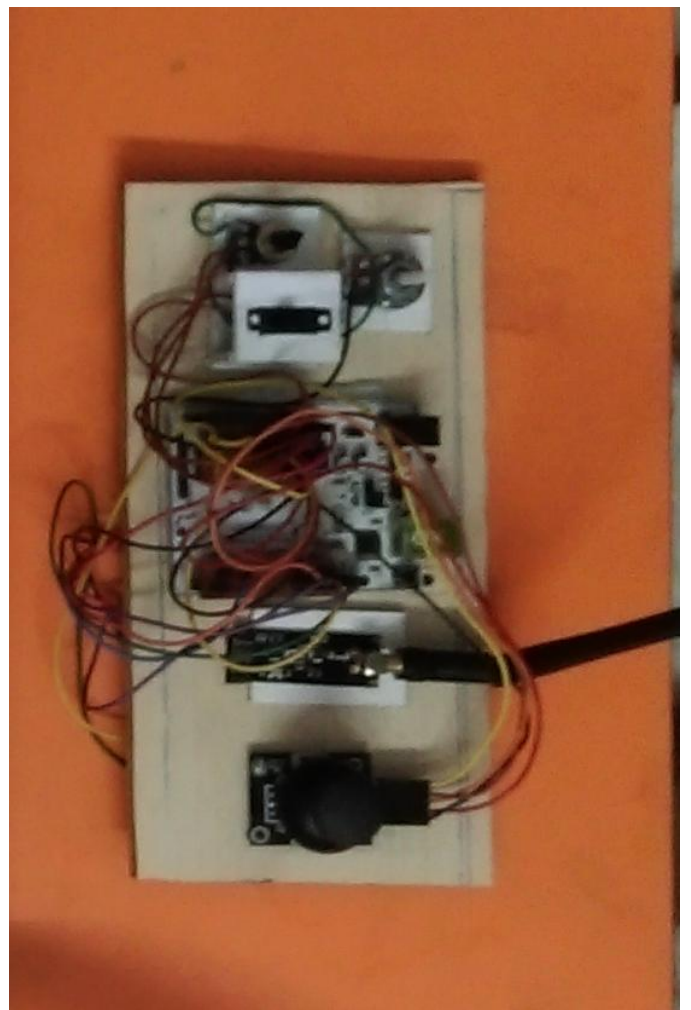

Fig.7 nRF controller for quad copter

\section{RESULT}

The quad copter which has completed its design with the weight of approximately one kilogram has got the payload of half kilogram. That is this quad copter can carry the load weighing up to half kilogram and then it can deliver the load to destination with the help of camera which can be controlled by servo motor and it gives the visual of destination as well as docking.

\section{CONCLUSION}

In this paper, an outline of a quad copter design and its implementation is presented. When designing a control system for a stable flight such as quad copter, we can see on practice the influence of various types of controllers on the overall stability of the inherently unstable aerial goods as well as in different areas like surveillance, military operations, fire sensing and some important areas having many complexities.

\section{REFERENCES}

[1] Robust Take-Off for a Quadrotor Vehicle; D. Cabecinhas, R. Naldi, L. Marconi, C. Silvestre, and R. Cunha.

[2] Landing a VTOL Unmanned Aerial Vehicle on a Moving Platform Using Optical Flow; Bruno H’eriss'e, Tarek Hamel, Robert Mahony, and Franc, ois-Xavier Russotto.

[3] System Identification, Estimation and Control for a Cost Effective Open-Source Quadcopter; Inkyu Sa and Peter Corke.

[4] Quadcopter Design and Implementation as a Multidisciplinary Engineering Course; Igor Gaponov Department of Mechanical Engineering Korea University of Technology and Education Cheonan City, South Korea; Anastasia Razinkova Department of Electrical Engineering Korea University of Technology and Education Cheonan City, South Korea.

[5] Designing a Spatially Aware, Automated Quadcopter Using an Android Control System; Carolyn Pearce, Margaret Guckenberg, Bobby Holden, Andrew Leach, Ryan Hughes, Connie Xie, Meredith Hassett, Andrew Adderley, Laura E. Barnes, Mark Sherriff, and Gregory C. Lewin University of Virginia.

[6] Altitude Controller Design For Multi-Copter UAV; Zaki Mustapa, Shakir Saat, S. H. Husin, Norafizah Abas Faculty of Electronic and Computer Engineering University Teknikal Malaysia Melaka, Malaysia. 